\title{
Evaluation of the Vertical Method of Toothbrushing
}

\author{
by Richard A. Shick, D.D.s., M.S., Flint, Micbigan anv \\ Major M. Ash, JR.," D.D.s., M.s., Ann Arbor, Micbigan
}

$\mathrm{T}$ HE relative effectiveness of the vertical method of toothbrushing in preventing or removing the soft accretions on the surfaces of the teeth has never been conclusively studied. The objective of this study was to evaluate the effectiveness of the vertical method of brushing, using a toothbrush with an adjustable head, with the well known roll-method of brushing, using a "standard design" toothbrush.

REVIEW OF THE LLTERATURE

Different methods have been used to evaluate oral hygiene and the effectiveness of brushing teeth. A frequently used procedure is the application of solutions to disclose the presence of debris or dental plaque on the teeth. Chawla, Nanda and Mathur, ${ }^{4}$ Curtis, McCall and Overaa, ${ }^{5}$ and Ramfjord, ${ }^{20}$ used bismark brown in their disclosing solutions. Ramfjord, ${ }^{20}$ in an epidemiological survey, observed the amount of stained dental plaques on the teeth and scored the individual teeth as $0,1,2$ or 3 . Greene $^{8}$ used a similar scoring index to record the amount of debris present on the surfaces of teeth without the use of a disclosing solution. The indexes of Ramfjord ${ }^{20}$ and Greene ${ }^{8}$ may be used for epidemiological studies but they are not sensitive enough to record the small differences in the presence of dental plaque which might be expected when measuring the effectiveness of toothbrushing.

The roll-method (sweeping motion) is "rather universally used"2 and often is advocated. ${ }^{2,5,6,9,13}$ The advocates of the roll-method agree that the "standard design" of toothbrush is used effectively and efficiently with this method of brushing. Thus, the roll-method using the "standard

*Associate Professor of Periodontics at the School of Dentistry of The University of Michigan. design" toothbrush was selected to be used as a basis of comparison with the vertical method of brushing (scrubbing motion) using a toothbrush with an adjustable head. A personal preference has been expressed by many writers for natural bristles; $1,3,6$, 9,13 however, there is no scientific or clinical evidence to indicate that either natural or nylon bristles are superior in removing plaques from the teeth. The only significant evidence presented for a particular type of bristle are the results of the laboratory studies $11,12,19,22$ which indicated that nylon bristles were more uniform in diameter and stiffness than natural bristles. Thus, nylon bristles were used in this study that is being reported.

While commenting on the selection of groups with a limited number of subjects, Hill10 stated, "with small numbers it will be wise to ensure equality, or near equality, by design." By design, Curtis, McCall and Overaa ${ }^{5}$ grouped all the subjects in their study on the basis of stained teeth and the presence of gingivitis in order to have each group represent as equal as possible a typical sample of the entire body of subjects. Several other investigators ${ }^{7,16,18,21}$ have attempted to equate their groups in all respects relevant to their studies. Thus, the method of choice for selecting the subjects or forming groups for studying toothbrushing should be "by design" rather than by random sampling.

The effectiveness of various methods of toothbrushing and the use of various toothbrushes were reported in six studies. $15,17,18,21$ In these studies, a particular method of brushing or a particular toothbrush has been recommended. However, since none of the methods of scoring were analyzed for reproducibility, and none of the studies utilized precise criteria for scoring every tooth in the mouth, one may conclude that there is no substantiation for 


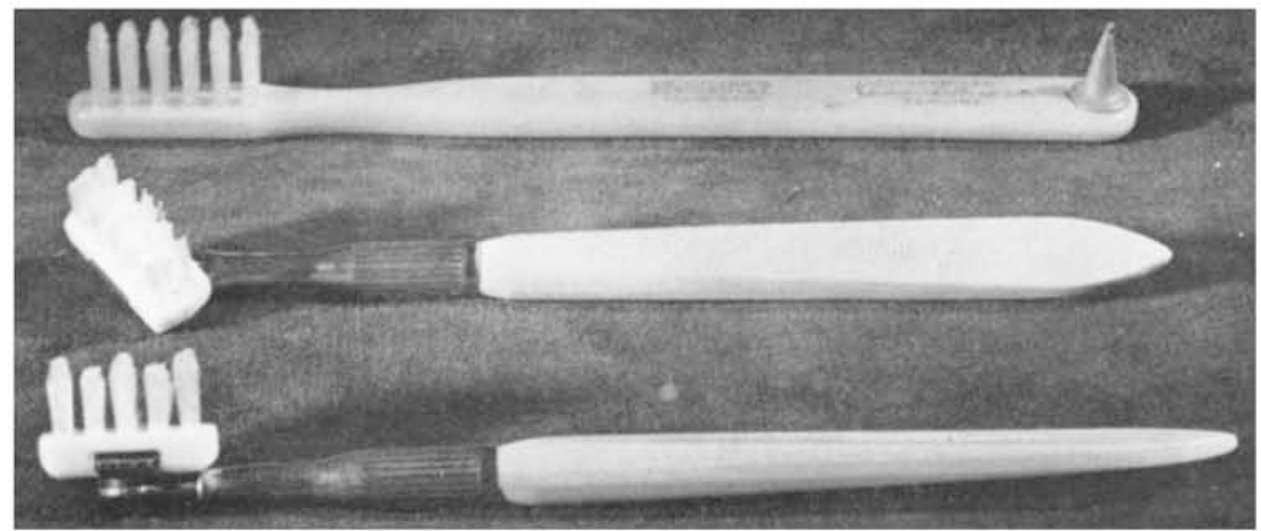

Fig. 1. Standard Design Toothbrush and Two Toothbrushes With an Adjustable Head.

recommending a particular toothbrush or method of brushing. Also, none of these studies were concerned with the vertical method of brushing.

\section{MATERIALS AND METHODS}

Two different types of toothbrushes were utilized for this study," a "standard design" (SD) and an adjustable head $(\mathrm{AH})$ (Figure 1). In order to determine the relative effectiveness of the vertical method of brushing with an $\mathrm{AH}$ toothbrush, the roll-method of brushing with an SD toothbrush was used as a basis for comparison. The SD toothbrush was selected because it has been recommended frequently for use with the roll-method of brushing. The AH toothbrush was selected because it was one of the few brushes specifically designed to be used with the vertical method of brushing.

The SD toothbrush had a solid straight handle and the bristles were arranged in two rows of six tufts. The SD toothbrush was accepted as effective for cleaning all surfaces of the teeth. The AH toothbrush had the head mounted on a wire loop so that one could rotate it $360^{\circ}$. The handle of the toothbrush could be turned to adjust the frictional tension on the head of the

"The "standard design" brush was supplied by the Dr. Butler Company and the toothbrush with an adjustable head was provided by the Clean-Be-Tween Corporation. All bristles were hard nylon. brush in order to hold it in any desired position. It also could be loosened so that the head could be replaced. The adjustable head facilitated the brushing of the facial (buccal and labial) and lingual surfaces of the teeth by short up-and-down strokes called the vertical method of brushing. The $\mathrm{AH}$ toothbrush had the bristles arranged in three rows of five tufts. When viewing this brush from the side, the tufts were found to be alternately long and short (Figure 1). This design was utilized to make it possible for the longer bristles to enter and clean the interproximal areas. All toothbrushes utilized in this study had "hard" nylon bristles.

Male Junior dental students were selected for this study. The subjects were similar in age, motivation, educational status, concepts of oral hygiene and gingival health. No students were used who had periodontitis, acute or recurrent necrotizing gingivitis, hyperplastic gingivitis or loss of interproximal tissue. This selection of subjects was utilized to minimize the variable factors not directly controlled by the study.

Methods of Tootbbrusbing. When using the roll-method of brushing, the sides of the bristles are placed horizontal with the long axis of the teeth and pressed against the attached gingiva with sufficient force to blanch the tissues momentarily. By rolling the wrist, the tips of the bristles are 


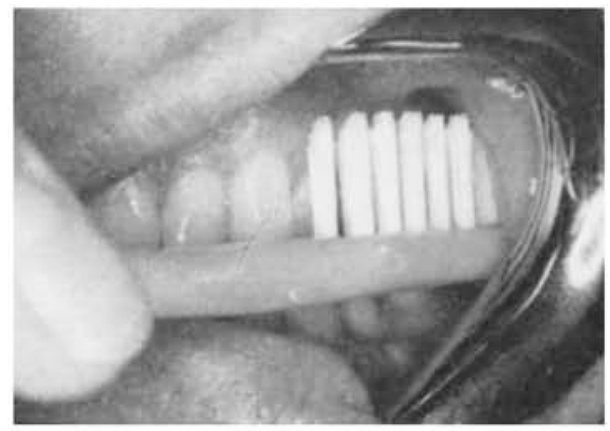

Fir. 2A. Bristles are horizontal with the long axis of the teeth.

moved slowly and firmly off the gingiva and lengthwise over the surfaces of the teeth. Briefly stated, this method brushes down on the upper teeth and up on the lower teeth (Figure 2).

When using the vertical method of brushing, the head of the AH toothbrush is rotated to an angle of approximately $45^{\circ}$ with the handle (Figure 1). With this adjustment of the head of the toothbrush, the facial surfaces of the teeth on one side of the mouth are brushed, also the mandibular lingual surfaces of the same side and the maxillary lingual surfaces on the opposite side of the mouth. The anterior teeth are positioned end-to-end and the bristles are placed at right angles to the facial surfaces of the teeth. The bristles then are moved up and down, brushing both maxillary and mandibular teeth with the ends of the bristles while massaging the gums with the sides of the bristles. When brushing the lingual surfaces of the teeth, the mouth is opened wide. In order to brush the rest of the teeth, the head of the brush is rotated so that it forms a $45^{\circ}$ angle with the handle in a reverse manner to the previous setting and the remaining facial and lingual surfaces are brushed as previously described (Figure 4). If necessary, the head of the toothbrush may be turned at a right angle to the handle to facilitate the brushing of the facial and lingual surfaces of the anterior teeth.

It was emphasized during toothbrushing instructions that the sides of the bristles, not the ends, were to be used to massage the gums.

Scoring Plaques. Preliminary observations showed that none of the scoring indexes described in the literature were sufficiently sensitive to measure the small differences which might occur in a study of this nature. By modifying the epidemiological scoring indexes of Ramfjord ${ }^{20}$ and Greene, ${ }^{8}$ an index was developed to score small changes in the amount of plaque present on the gingival halves of the coronal surfaces of the teeth. Methods of scoring dental plaques cannot be considered reliable unless the results of scoring the same plaques can be reproduced within the limits of variability of the scoring index. In order to evaluate the reproducibility of

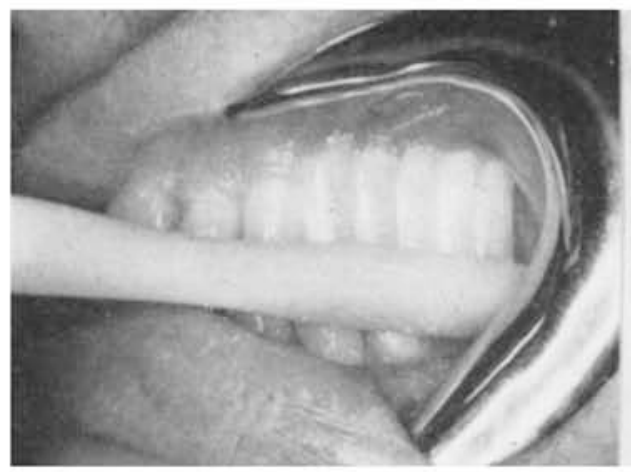

The tissues are blanched momentarily.

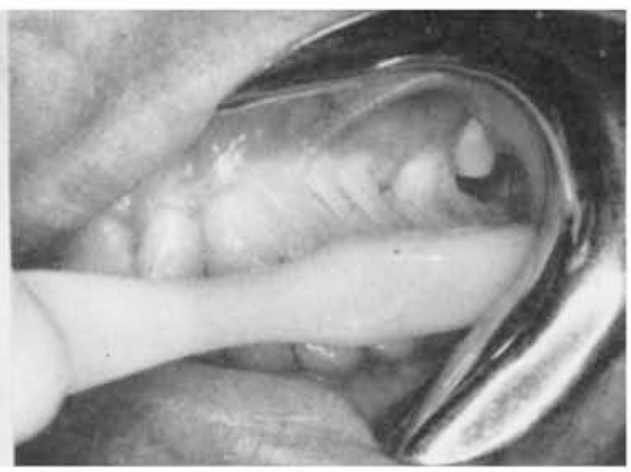
Bristles move lengthwise over the surfaces of
the teeth.

Figure $2 \mathrm{~B}$ 


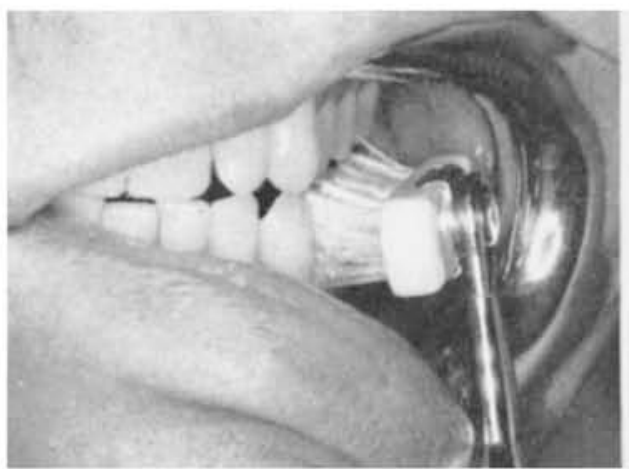

(left) Brush down.

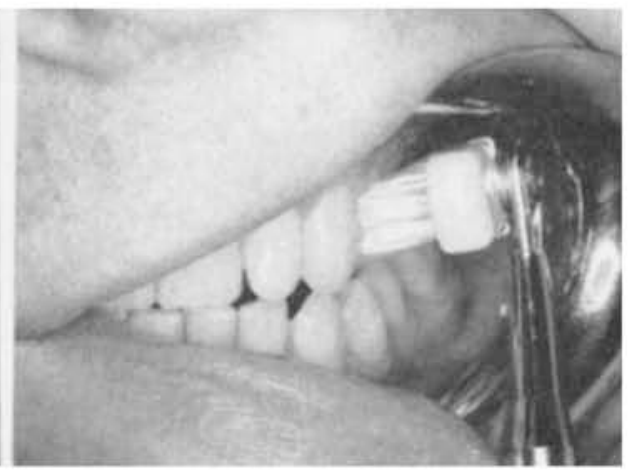

(right) Brush up.

Figure 3

the scoring index used in this study, four subjects were scored three times in the same morning. Cotton applicators were soaked in disclosing solution and the solution first was applied to the mandibular and then the maxillary teeth, covering one quadrant at a time. Each student was instructed to rinse his mouth thoroughly two times with water. Each tooth received a facial and a lingual score. The first scores for the individual teeth of each subject were compared with the second scores, and the second scores were compared with the third scores. These results indicated that the degree of variation of the three scorings was insignificant, and thus the scoring index was reproducible and reliable.

The amount of the surface covered by dental plaque was scored as $0,1,2$ or 3 . "Dental plaque" was accepted as any soft accretion on the surface of a tooth that would retain disclosing solution. The absence of dental plaque on the gingival half of the facial surface of a tooth was scored as 0 ; the presence of dental plaque covering less than one-third of the gingival half of the facial surface was scored as 1 ; the presence of dental plaque covering onethird or less than two-thirds of the gingival half of the facial surface was scored as 2; and the presence of dental plaque covering two-thirds or more of the facial surface was scored as 3 (Figure 5). The same scoring procedure was used for the lingual surfaces of the teeth. Thus, each tooth received a facial and a lingual score.

In order to convert the scores of dental plaques into a percentage-score (PS), the individual scores $(\mathrm{S})$ of each tooth were added to get the total score (TS) of the

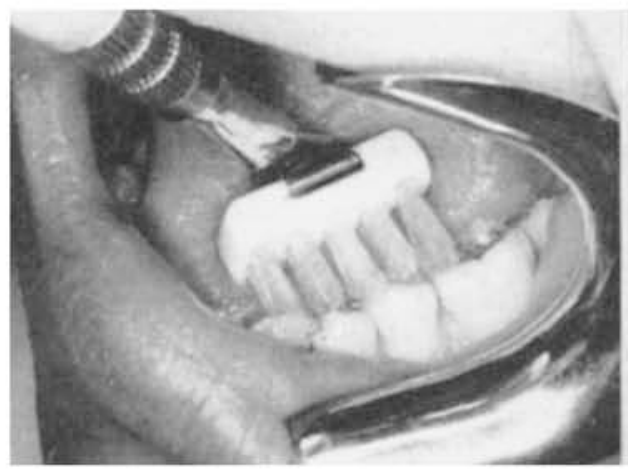

Brush lingual surfaces of mandibular teeth.

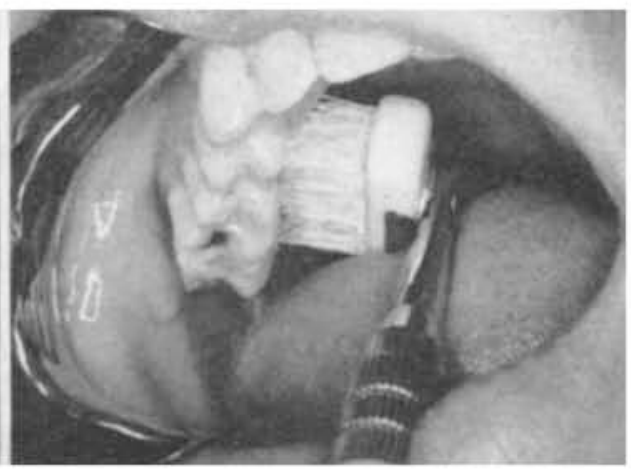

Brush lingual surfaces of maxillary teeth.

Figure 4 
Summary of Procedures

1. A scoring index was developed and standardized to measure the amount of dental plaque on the coronal surfaces of the teeth.

\begin{tabular}{|c|c|c|c|c|c|c|c|c|}
\hline \multicolumn{3}{|c|}{$\begin{array}{l}\text { 2. Clinical procedure- } \\
80 \text { subjects } \\
\text { scored for } \\
\text { dental plaque } \\
\text { and then } \\
\text { provided with } \\
\text { a prophylaxis. }\end{array}$} & $1 \mathrm{wk}$. & $\begin{array}{l}\text { Rescored } \\
\text { for } \\
\text { dental } \\
\text { plaque. }\end{array}$ & $3 \mathrm{wks}$ & $\begin{array}{l}\text { Rescored } \\
\text { for dental } \\
\text { plaque. } \\
\text { Four equal } \\
\text { groups were } \\
\text { formed. }\end{array}$ & \multicolumn{2}{|c|}{$\begin{array}{l}\text { (A) } 20 \text { subjects } \\
\text { (B) } 20 \text { subjects } \\
\text { (C) } 20 \text { subjects } \\
\text { (D) } 20 \text { subjects }\end{array}$} \\
\hline 3. & (A) & $\begin{array}{l}\text { Teeth } \\
\text { Polished }\end{array}$ & $1 \mathrm{wk}$. & $\begin{array}{l}\text { TBI-Vert. } \\
\text { AH-Brush }\end{array}$ & $3 \mathrm{wks}$. & $\begin{array}{l}\text { Scored } \\
\text { TBI-Vert. } \\
\text { AH-Brush }\end{array}$ & $4 \mathrm{wks}$. & $\begin{array}{l}\text { Final } \\
\text { Scoring }\end{array}$ \\
\hline & (B) & $\begin{array}{l}\text { Teeth } \\
\text { Polished }\end{array}$ & I wk. & $\begin{array}{l}\text { TBI-Roll } \\
\text { SD-Brush }\end{array}$ & $3 \mathrm{wks}$. & $\begin{array}{l}\text { Scored } \\
\text { TBI-Roll } \\
\text { AH-Brush }\end{array}$ & $4 \mathrm{wks}$. & $\begin{array}{l}\text { Final } \\
\text { Scoring }\end{array}$ \\
\hline & (C) & $\begin{array}{l}\text { Teeth } \\
\text { Polished }\end{array}$ & $1 \mathrm{wk}$. & $\begin{array}{l}\text { TBI-Roll } \\
\text { SD-Brush }\end{array}$ & $3 \mathrm{wks}$. & $\begin{array}{l}\text { Scored } \\
\text { TBI-Roll } \\
\text { SD-Brush }\end{array}$ & $4 \mathrm{wks}$. & $\begin{array}{l}\text { Final } \\
\text { Scoring }\end{array}$ \\
\hline & (D) & $\begin{array}{l}\text { Teeth } \\
\text { Polished }\end{array}$ & $1 \mathrm{wk}$. & $\begin{array}{l}\text { TBI-Vert. } \\
\text { AH-Brush }\end{array}$ & $3 \mathrm{wks}$. & $\begin{array}{l}\text { Scored } \\
\text { TBI-Vert. } \\
\text { SD-Brush }\end{array}$ & 4 wks. & $\begin{array}{l}\text { Final } \\
\text { Scoring }\end{array}$ \\
\hline & & & & $\begin{array}{l}\text { TBI } \\
\text { Vert. } \\
\text { Roll } \\
\text { AH-Brush } \\
\text { SD-Brush }\end{array}$ & \multicolumn{4}{|c|}{$\begin{array}{l}\text { - Instructions in toothbrushing } \\
\text { - Vertical method of brushing } \\
\text { - Roll-method of brushing } \\
\text { - Toothbrush with an adjustable head } \\
\text { - "Standard design" of toothbrush }\end{array}$} \\
\hline
\end{tabular}

4. Data were statistically analyzed.

dental plaques for each subject. The total score was divided by the highest possible theoretical score (HTS) and this quotient was multiplied by 100 to arrive at a percentage-score for each individual.

$$
\mathrm{PS}=\frac{\mathrm{TS}}{\mathrm{HTS}} \times 100
$$

The percentage-scores thus were comparable for all individuals regardless of the number of teeth present in the mouth of a subject.

The manner of grouping and alteration of the variables made it possible to compare the two different methods of brushing or the two different types of toothbrushes in relation to their effectiveness for preventing or removing the dental plaque from the coronal surfaces of the teeth.

Clinical Procedures. After the formation of the groups, all of the subjects' teeth were polished with a rubber cup and pumice to remove the dental plaques and reduce all of the scores to 0 . Each subject received two new toothbrushes and a three minute intra-oral demonstration of toothbrushing. The method of stating instructions in toothbrushing was practiced and standardized prior to the demonstrations. All of the subjects in each group were given comparable instructions for approximately the same length of time. They were instructed to use only the method of brushing and toothbrushes that were provided. Four weeks from the date the teeth were polished, the dental plaques again were scored. At this time, Group B was changed to the AH toothbrush and Group $\mathrm{D}$ changed to the $\mathrm{SD}$ toothbrush while Groups $A$ and $C$ continued with the same type of brush and all groups were given two new toothbrushes. All groups were given reinstructions in the same method of brushing previously employed. The change in toothbrushes for two of the groups provided a means of comparing the two types of toothbrushes using either method of brushing and the two methods of brushing using either type of toothbrush. Four weeks 
Method of Scoring the Presence or Abscence of Dental Plaque

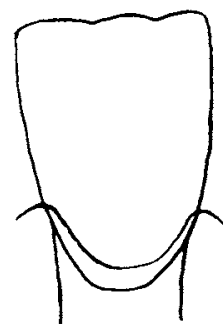

Score - 0

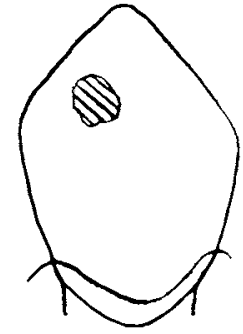

Score - 0

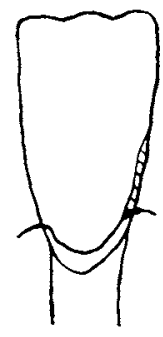

Score - 1

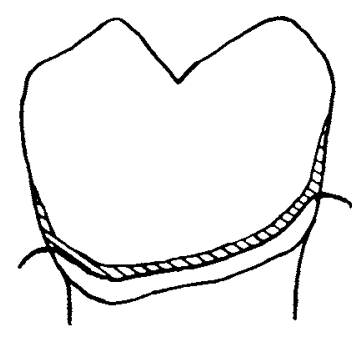

Score - 1

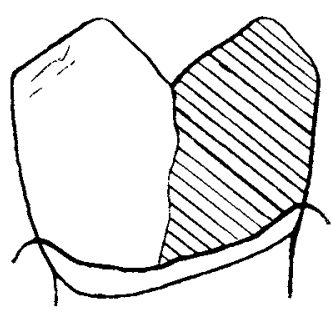

Score -2

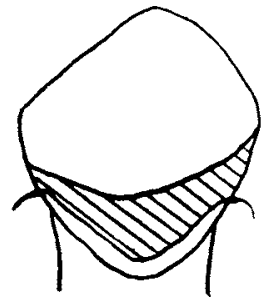

Score -2

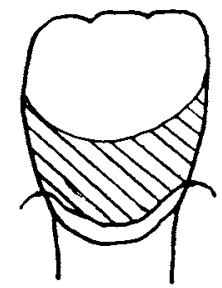

Score -3

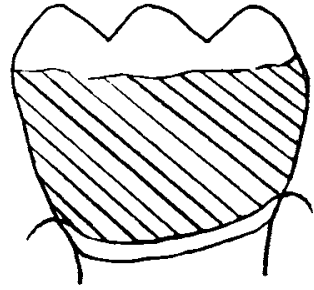

Score -3

Figure 5

from the previous scoring, the subjects were scored for dental plaques for the final time.

Statistical Analysis of Data. The " $\mathrm{t}$ " test was used to analyze the between-group and within-group differences. Formula 1 was used to analyze the differences between the groups and Formula 2 was used to analyze the differences within the groups.

$$
\begin{array}{ll}
\text { (1) } t= & \frac{\bar{M}_{1}-\overline{M_{2}}}{s} \quad s^{2}=\frac{s x_{1}^{2}+s x_{2}^{2}}{N_{1}+N_{2}-2} \\
(2)+=\frac{\bar{D}}{N_{1}} & s^{2}=\frac{N \times s D^{2}-(S D)^{2}}{N \times(N-1)} \\
& \sqrt{N}
\end{array}
$$

RESULTS

The base-line mean dental plaque scores were calculated (from the first three scorings) for each of the four groups and also the mean dental plaque scores for the fourth and fifth scorings of each group (Table 1).

$$
\text { TABLE I }
$$

Base Line, Fourth and Fifth Scoring-Means for Each Group

\begin{tabular}{cccc}
\hline Groups & $\begin{array}{c}\text { Base- Line Means } \\
(\text { Avg. of first } \\
\text { three scorings })\end{array}$ & $\begin{array}{c}\text { Means for } \\
\text { 4th Scoring }\end{array}$ & $\begin{array}{c}\text { Means for } \\
\text { Stb Scoring }\end{array}$ \\
\hline A & 42.25 & 35.80 & 35.35 \\
B & 42.60 & 35.65 & 37.25 \\
C & 42.20 & 34.85 & 34.45 \\
D & 42.25 & 35.00 & 35.95 \\
\hline
\end{tabular}

Results of the calculations using the " $t$ " test to analyze the differenecs between the groups and within the groups from the fourth to fifth scorings indicated there was no statistically significant differences at the five per cent level of confidence. However, when comparing the baseline means with 
the means of the fourth and fifth scorings, there was a statistically significant difference at the one per cent level of confidence.

Since Ramfjord, ${ }^{20}$ when doing an epidemiological study, selected six teeth to represent all the teeth of a subject, it was decided to compare the plaque scores using these six teeth with the plaque scores when using all the teeth. The results indicated there was no statistically significant difference in the scores of the dental plaque for the first three scorings when using six teeth or all the teeth of a subject.

\section{DISCUSSION}

The analysis of the differences between the four groups, using the means of the 4 th and 5 th scorings or analyzing the differences within the groups for these same means, produced no statistically significant differences at the five per cent level of confidence when using the " $t$ " test. These results indicate that the vertical method of brushing with a toothbrush that had an adjustable head, was an effective way to prevent or remove dental plaque when compared with the roll-method using a "standard design" toothbrush. The results of this study also indicate that there was no difference in the prevention or removal of dental plaque when (1) using the rollmethod of brushing with either the SD or $\mathrm{AH}$ brush, (2) using the vertical method of brushing with either the SD or $\mathrm{AH}$ brush, (3) using the SD brush with either the vertical or roll-method of brushing and (4) using the AH brush with either the vertical or roll-method of brushing.

The analysis of the differences within the groups, when comparing the four baseline means with the means of the fourth and fifth scorings, demonstrated a statistically significant difference at the one per cent level of confidence in every comparison. These results indicate that after new toothbrushes and instructions were given, all of the groups, regardless of the method of brushing or toothbrush utilized, showed a statistically significant improvement in the effectiveness of preventing or remov- ing dental plaque on the gingival half of the coronal surfaces of the teeth. Thus, the new toothbrushes and instructions appear to be more effective, in obtaining favorable results, than the type of toothbrush or method of brushing.

The preliminary testing of the index for scoring dental plaque indicated that this method of measuring the presence or absence of dental plaque on the gingival half of the coronal surfaces of the teeth was both reliable and reproducible when measuring the same plaques. The weakest aspect of this scoring index was the differentiation between a score of 0 or 1 . It is possible that the addition of another division between 0 and 1 would make the index more sensitive in scoring the small changes in the presence of plaques. With such a modification, it should be possible for a study to determine if instructions in the various methods of toothbrushing with different toothbrushes would produce not only a statistically significant improvement but also a biologically significant improvement.

Ramfjord's ${ }^{20}$ selection of six teeth to represent the entire mouth when scoring dental plaques appears to be an accurate representative selection. Thus, the use of these six teeth instead of all a subject's teeth, would facilitate using large numbers of subjects when doing a study related to the presence or absence of dental plaque.

\section{SUMMARY}

Eighty Junior dental students were divided into four groups which were as nearly alike as possible for the purpose of this study. Using these groups, an evaluation was made of the vertical method of brushing using a toothbrush with an adjustable head. The roll-method of brushing with a "standard design" toothbrush was selected as a basis for comparison. The degree to which stained dental plaques covered the gingival halves of the coronal surfaces of the teeth was used as an index for measuring the effectiveness of the toothbrushing. The " $t$ " test was utilized 
to compare the results before and after the introduction of toothbrushes and instructions and also to compare the two methods of brushing with the two different toothbrushes.

\section{CONCLUSIONS}

Within the limits of the design of this study, it was possible to make the following conclusions:

1. The vertical method of brushing with a toothbrush that had an adjustable head was equally as effective for removing or preventing the dental plaque as the rollmethod using a "standard design" of toothbrush.

2. The "standard design" of toothbrush and the toothbrush with an adjustable head seem to be equally effective with either the roll or vertical method of brushing for removing or preventing dental plaque.

3. The scoring index utilized appears to be a reliable method for measuring the effectiveness of toothbrushing in respect to the presence of dental plaque.

4. The instructions with a new toothbrush appear to be of greater importance for removing or preventing dental plaque than the use of a particular type of toothbrush or method of brushing.

5. The mean plaque scores of six teeth were found to be representative of the mean plaque scores when using all of the teeth.

\section{BIBLIOGRAPHY}

1. Bell, D. G. Teaching home care to the patient. J. Periodont., 19:140-3, Oct. 1948 .

2. Bunting, R. W. Oral hygiene. 3 rd ed., Philadelphia, Lea and Febiger, 1957. 334 p. (p. 27-8)

3. Chaters, W. J. Immunizing hard and soft tissues to infection by correct stimulation with the toothbrush. South. Calif. S. Dent. A. I., 3:1037-41, Oct. 1936.

4. Chawla, T. N., Nanda, R. S., and Mathur, M. N. Bacterial plaque and its relation to periodontal disease. All-India Dent. A. J, 31:121-5, Aug. 1959.
5. Curtis, G. H., et al. Clinical study of the effectiveness of the roll and Charter's methods of brushing teeth. J. Periodont., 28:277-80, Oct. 1957.

6. Davis, H. C. The toothbrush. Dent. Practitioner, 1:193-200, Feb. 1951.

7. Goldman, H. M. Effect of single and multiple tooth brushing in the cleansing of the normal and periodontally involved dentition. Oral Surg., Oral Med. and Oral Path., 9:203-4, Feb. 1956.

8. Greene, J. C. Periodontal disease in India: Report of an epidemiological study. J. Dent. Res., 39:302-12, March-April 1960.

9. Hard, Dorothy G. Dental scientist suggests watching mirror while brushing teeth. Ark. S. Dent. A. J., 23:18, Sept. 1952.

10. Hill, A. B. Principles of medical statistics. 6th ed., New York, Oxford University Press, 1956. IX + 314 p. (p. 239-41)

11. Hine, M. K. Jr. Variation in toothbrush textures. Am. Dent. A. J., 46:536-9, May 1953.

12. Hine, M. K. The use of the toothbrush in the treatment of periodontitis. Am. Dent. A. J., 41:158-68, Aug. 1950 .

13. Hirschfeld, Isador. The why and how of toothbrushing. Am. Dent. A. J., 32:80-5, Jan. 1, 1945 .

14. Kimmelman, B. B., et al. Research in toothbrush design. Pa. S. Dent. J., 25:24-9, Apr. 1958.

15. Kimmelman, B. B., and Tassman, G. C. Research in designs of children's toothbrushes. J. Dent. Child., 27:60-4, 1st Quar. 1960.

16. Lovdal, A. L., Arnulf, Arno, and Waerhaug, Jens. Incidence of clinical manifestations of periodontal disease in light of oral hygiene and calculus formation. Am. Dent. A. J., 56:21-33, Jan. 1958.

17. Mason, W. I. A more efficient toothbrush head. Canad. Dent. A. J., 15:327-9, June 1949.

18. Maurice, C. G., and Wallace, D. A. Toothbrush effectiveness: relative cleaning ability of four toothbrushes of different design. Ill. Dent. J., 26:286-92, May 1957 .

19. McCauley, H. B. Toothbrushes, toothbrush materials and design. Am. Dent. A. J., 33:283-93, Mar. 1946.

20. Ramfjord, Sigurd. Indices for prevalence and incidence of periodontal disease. J. Periodont., 30:51-9, Jan. 1959.

21. Reese, J. W. Toothbrushing for orthodontic patients. Ann Arbor, University of Michigan, School of Dentistry, 1959. 55 p. typed thesis.

22. Swartz, Marjorie L., Phillips, R. W., and Hine, M. K. Effect of certain factors upon toothbrush bristle stiffness. J. Periodont., 27:96-101, Apr. 1956. 